\title{
Effects of prior social experience on agonistic responding by Siamese fighting fish (Betta splendens)
}

\author{
NANCY WOODARD CAIN, REID ANDERSON, LORI STEIN, and CYNTHIA JESSEN \\ Bucknell University, Lewisburg, Pennsylvania 17837
}

\begin{abstract}
The role of prior social experience in male Betta agonistic behavior was investigated in two experiments. Fish were either paired with an opponent until dominance was established naturally (Experiment 1) or given prior combat experience with a known aggressive or nonaggressive opponent (Experiment 2). The effects of prior social experience were then observed both in additional paired encounters and in some noncombat agonistic situations. The pairedencounter data indicate that Betta attack aggressive opponents more than nonaggressive ones and rarely attack opponents once they have submitted. In contrast to attacking, these fish do not show a significant decrease in displays once their opponent has submitted, nor do they display differentially to aggressive and nonaggressive opponents. The noncombat agonistic-situation data indicate that prior social experience affects both the preference of fish for viewing other conspecifics and the waning of display behaviors. These results suggest that submissive and nonaggressive behaviors can inhibit attacks but not displays by Betta, and they emphasize the necessity of controlling for prior social experience when studying aggressive behavior.
\end{abstract}

The importance of experiential changes in agonistic behavior has been recognized for some time. Indeed, experiential changes are central to both reinforcement interpretations (see Baenninger, 1974, for a review) and less traditional explanations (e.g., Leshner \& Nock, 1976) of the motivation underlying agonistic responding. Much of the research on prior social experience and agonistic responding has been done with Betta. Betta are particularly well suited for investigations of aggressive behavior for several reasons. They engage in sequences of agonistic responding in which threats (displays) and attacks can be easily detected, and various components of the agonistic sequence are modifiable through learning.

The presence of considerable individual differences in Betta aggressive behavior has led many investigators to speculate that this variability may be the result of wide differences in prior social experience. Prior social experience has been operationally defined in a number of ways, including dominant and subordinate roles resulting from paired encounters (Baenninger, 1968, 1970; Lobb \& McCain, 1976; Meliska, Meliska, Hoyenga, Hoyenga, \& Ward, 1975), prior visual experience with a conspecific (Meliska \& Meliska, 1976; Miley \& Burack, 1977), and visual and combat experience with responsive or passive conspecifics and nonconspecifics (Johnson \& Johnson, 1973).

Once differences in social experience have been established, these differences appear to account for

The authors thank D. Candland, O. Floody, and A. Leshner for critical comments on an earlier version of this manuscript and P. McIntosh, S. Slike, and L. Trescott for technical assistance. varying levels of aggressive motivation. For example, dominant fish perform operant tasks for mirror reinforcement at a high level, whereas subordinate fish appear to be less inclined to view their mirror images (Baenninger, 1970). Additionally, subordinate fish cease to attack and display to their opponents once they have submitted (Baenninger, 1968; Lobb \& McCain, 1976). The behavior of dominant fish in subsequent paired encounters, however, is not so clear. Baenninger (1968) has presented data which indicate that submissive behaviors have little suppressive effect on attacks and displays by dominant fish. On the other hand, Dore, Lefebvre, and Ducharme (1978) recently have questioned these data and have suggested that visual appeasement signals do, indeed, prevent extensive physical damage in aggressive encounters.

Thus, there is only indirect or conflicting evidence concerning the effects of prior social experience on Betta agonistic responding. The following experiments are a more direct test of some of these effects. In Experiment 1, the behavior of dominant and subordinate pairs of fish was observed both before and after the establishment of dominance. Viewing preferences of these fish were then determined in a threealternative choice situation involving views of an aggressive conspecific, a nonaggressive conspecific, or no visual stimulus.

In Experiment 2, fish were given combat experience with either a responsive (aggressive) or passive (nonaggressive) conspecific prior to $96 \mathrm{~h}$ of continual mirror exposure. Mirror exposure was chosen because it is one experimental situation that is often 
used to study the waning of Betta aggressive behavior. Following mirror exposure, fish were again combat-tested with an aggressive or nonaggressive conspecific.

\section{EXPERIMENT 1}

\section{Method}

Subjects. Twenty-eight adult male Siamese fighting fish (Betta splendens) were obtained from a local supplier. They were housed individually in 1-liter glass bowls and were visually isolated from one another for the duration of testing. The water temperature was maintained between $21^{\circ}$ and $24^{\circ} \mathrm{C}$.

Apparatus. During paired encounters, fish were netted and placed into a 3.8-liter clear Plexiglas tank measuring $30.7 \times 12.8$ $\times 15.3 \mathrm{~cm}$. During preference testing, 2.2 -liter clear Plexiglas tanks, which measured $12.8 \times 15.3 \times 15.3 \mathrm{~cm}$ and housed the stimulus fish, were placed adjacent to each end of the tank.

Procedure. Twenty-six of the subjects were assigned randomly to 13 pairs. Two other fish served as stimulus fish during the preference tests. Each pair was placed together into a neutral tank and allowed to interact freely for $15 \mathrm{~min}$ or until one member of the pair made five successive attacks without its opponent fighting back. The water in the testing chamber was changed between tests. During these encounters, the experimenter scored the frequency of displays and attacks. All fish were paired with their same opponents once every week for a total of 7 consecutive weeks.

Six pairs of fish in which a dominant/subordinate relationship was evident were used as subjects during the preference testing portion of this experiment. ${ }^{1}$ One week following the final paired encounter, each fish was observed in a three-alternative choice situation. Three portions of a rectangular tank were assumed to represent choice areas. A smaller tank housing a live, nondisplaying male Betta was placed to the right end of the rectangular tank, and an identical tank housing a live, displaying male Betta was placed to the left. According to their position in the rectangular tank, the fish could choose to view either an aggressive or a nonaggressive conspecific. They could also remain in a neutral zone (center one-third of the rectangular tank) in between the two visual stimuli. Baseline preferences first were measured with neither stimulus present at the ends of the main tank. Each preference test lasted $10 \mathrm{~min}$, with the experimenter recording the position of the experimental fish at the end of each $30-\mathrm{sec}$ period. Thus, each fish had 20 baseline and 20 preference scores. All observations were made blind; that is, the experimenter had no knowledge of whether the fish being tested had been dominant or subordinate in the previous encounters.

\section{Results}

Paired encounters. Ten of the 13 pairs of male Betta established a clear dominant/subordinate relationship by the end of the seventh paired encounter; three of those relationships were established during the last paired encounter. Additionally, 9 of the 26 fish tested never attacked, although all 26 fish displayed during at least one encounter.

Individual $t$ tests for related measures (two-tailed) were performed on the display scores of dominant and subordinate pairs of fish for the encounters both before and after dominance was established. Results of these tests indicated that fish that became subordinate displayed less often than their dominant pair members both before dominance was established $[t(9)=4.87, p<.01]$ and during additional encoun- ters after dominance was established $[\mathrm{t}(6)=3.8$, $\mathrm{p}<.01]$. These data are presented in Figure la. As can be seen in the figure, eventually dominant fish did not show a significant decrease in their frequency of displaying once they became dominant $[\mathrm{t}(6)=1.31$, $p>$.05]. In contrast, only one of the seven fish that eventually became subordinate displayed in subsequent paired encounters once dominance had been established.

Although there was a significant difference in the number of displays between dominant and subordinate pair members, there was not a significant difference in the number of attacks they made prior to dominance establishment $[\mathrm{t}(9)<1$; see Figure $1 \mathrm{~b}]$. Once dominance was established, none of the subordinates ever attacked. Four of the seven fish that had become dominant attacked their subordinate pair members following dominance establishment, but only one of these fish attacked more than once.
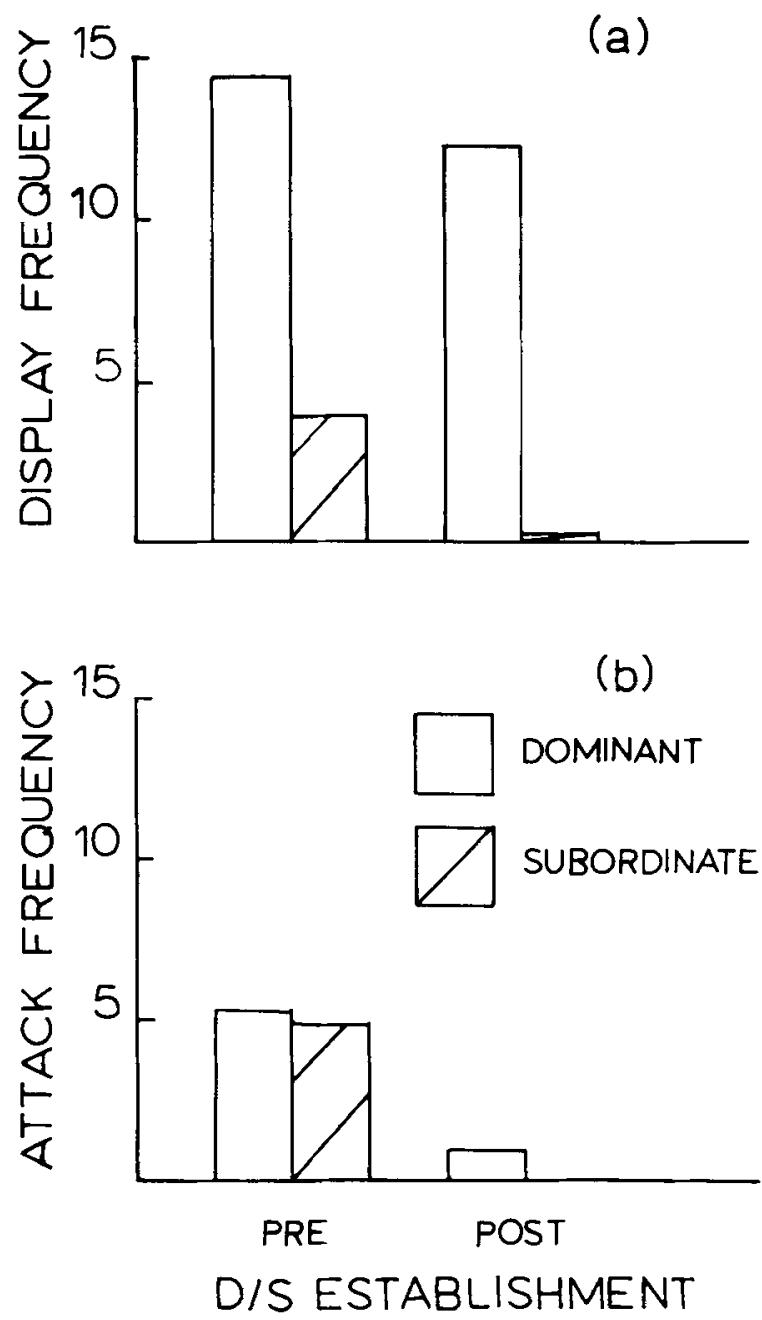

Figure 1. Mean number of displays (a) and attacks (b) per paired encounter for dominant and subordinate fish. Data are presented for paired encounters both before and after dominance was established. 


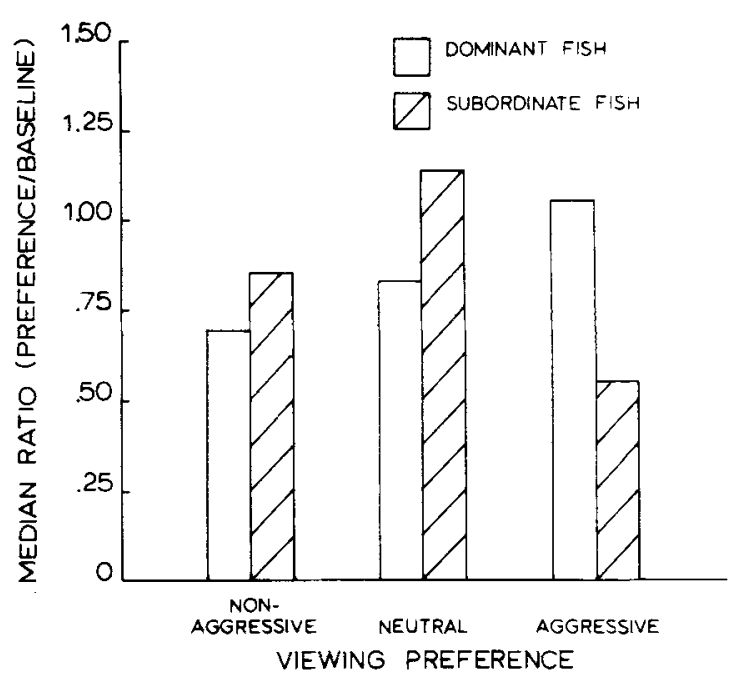

Figure 2. Position preferences of dominant and subordinate fish for viewing aggressive and nonaggressive conspecifics. A neutral preference indicates that fish were positioned in the center onethird of the tank. Each Betta preference score was divided by its baseline score, and the median ratio for dominant and subordinate experience groups is shown.

Preference test. Median ratios of the baseline to preference test scores were computed for each dominant and subordinate fish, and Wilcoxon matchedpairs signed rank tests were performed on these data. The results indicated that subordinate fish spent significantly more time in the center portion of the tank than did their dominant pair members $(T=3, p<.05$; see Figure 2). The median ratio of the baseline to preference scores for each of the three tank positions are presented in Figure 2. Although there was a ten-. dency for dominant fish to view the displaying stimulus more than the subordinate fish, this finding was not statistically significant $(T=6, p>.05)$. Additionally, no significant difference was found between the preference scores of the dominant and subordinate fish for viewing the nonaggressive stimulus fish $(\mathrm{T}=7, \mathrm{p}>.05)$.

\section{EXPERIMENT 2}

Experiment 1 investigated the effects of prior social experience, defined in this experiment as dominant and subordinate roles resulting from repeated paired encounters, on subsequent agonistic responding. The results demonstrated that, for dominant fish, the defeat of their opponent resulted in a decrease in the attack component of agonistic behavior but no change in the threat (display) component. Furthermore, once fish were defeated in a paired encounter, they were less inclined to view another conspecific than were their opponents. In Experiment 2, another type of experiential change in agonistic behavior was investigated. Fish were given combat experience with either a nonaggressive opponent, an aggressive opponent, or no combat experience at all. The effects of this experience on readiness to display to a mirror and on subsequent combat behaviors were evaluated.

\section{Method \\ Subjects. Twenty-three adult male Siamese fighting fish (Betta splendens) were obtained from a local supplier. Eighteen fish served as the experimental fish and five served as stimulus fish. All fish were housed individually in clear Plexiglas tanks and visually isolated from one another for the duration of the experi- ment. Water temperature was maintained between $21^{\circ}$ and $24^{\circ} \mathrm{C}$. \\ Apparatus. During paired encounters, fish were netted and transferred to a 28.5-liter glass aquarium measuring $41.4 \times 26$ $\times 21.5 \mathrm{~cm}$. During the viewing condition, the fish remained in their home tanks, 2.2-liter clear Plexiglas tanks that measured $12.8 \times 15.4 \times 15.4 \mathrm{~cm}$ and had flat glass mirrors attached to their ends.}

Procedure. The waning of the aggressive threat display was assessed following no prior combat experience, combat experience with a nonaggressive conspecific, or combat experience with an aggressive conspecific. Following acclimation to the laboratory for 6 days, 5 opponent fish were chosen from the 23 subjects. One, which never displayed and always maintained a pale coloration, was designated as the nonaggressive opponent. The other four fish were designated as the aggressive opponents; each had to meet the criteria of always performing a display and pursuing its opponent when confronted with another male conspecific. A particular aggressive opponent was used until it failed to meet the established criteria. It was then replaced by a new aggressive opponent.

Combat testing. The 18 experimental subjects were pretested for their ability to display during two successive $30-\mathrm{sec}$ mirror stimulation trials. They then were assigned randomly, six fish per group, to an aggressive-experience group, a nonaggressiveexperience group, or a no-combat-experience group. The fish in the aggressive-experience group were exposed three times to a 10-min combat encounter with an aggressive opponent, whereas the fish in the nonaggressive-experience group encountered the nonaggressive opponent for the same number and duration of encounters. The no-combat-experience group received no combat experience prior to the viewing condition. Each of the three 10-min paired encounters was separated by a 48 -h recovery period.

During these encounters, each fish was placed into a 28.5-liter glass aquarium which housed its appropriate opponent. The experimenter recorded the number and total duration of displays performed by each fish, as well as the number of attacks. At the end of the encounter, the subject was returned to its bome tank.

Mirror viewing. Following the third paired encounter with the same type of opponent, mirrors were attached to the end of each tank for 96 consecutive hours. The displays of each fish were observed for $10 \mathrm{~min}$ after the mirror was first put in place and then for $10 \mathrm{~min}$ at $8,24,48,72$, and $96 \mathrm{~h}$. All observations during this phase were made blind. The experimenter recorded the number of displays, the total duration of displays, and the total duration of avoidance behavior (time spent facing $180 \mathrm{deg}$ away from the mirror; cf. Peeke \& Peeke, 1970).

Combat retest. A final combat test for each fish in the aggressiveand nonaggressive-experience groups was performed immediately following the $96 \mathrm{~h}$ of mirror viewing. The same procedure, the same dependent measures, and the same opponents used in the previous three combat tests were employed.

\section{Results}

Mirror viewing. The duration of display and avoidance behaviors (facing $180 \mathrm{deg}$ away from the mirror) were subjected to ANOVAs with combat experience as an independent factor and the hours of mir- 


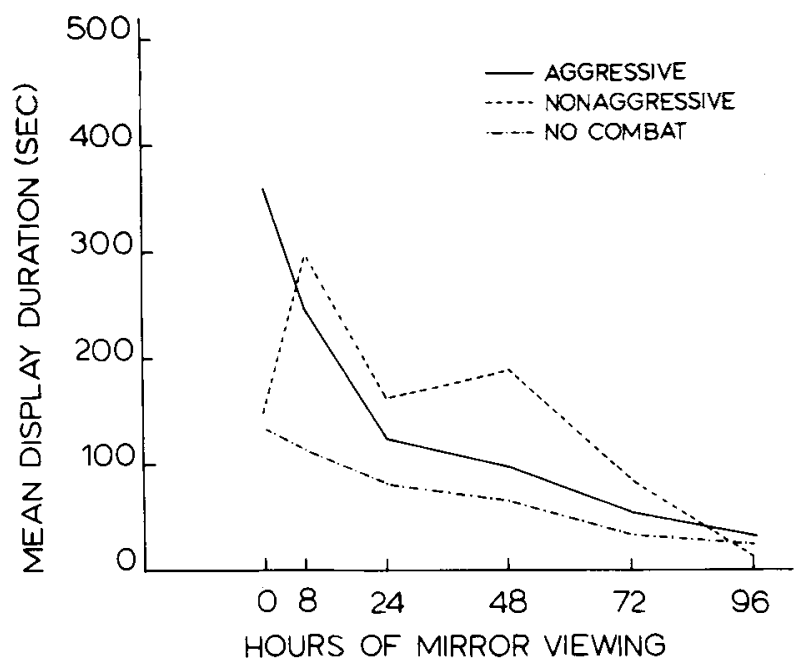

Figure 3. Mean duration of displays (in seconds) for aggressive-, nonaggressive-, and no-combat-experience groups over $96 \mathrm{~h}$ of mirror viewing.

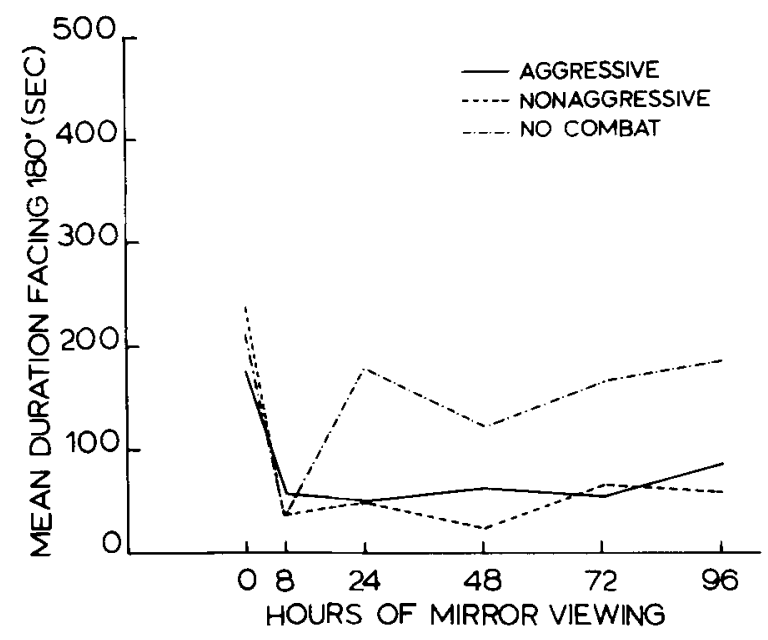

Figure 4. Mean duration of avoidance behaviors (seconds facing 180 deg away from their mirror reflections, for aggressive-, nonaggressive-, and no-combat-experience groups over $96 \mathrm{~h}$ of mirror viewing.

ror exposure as the repeated factor. ANOVA indicated that display durations decreased significantly with increased mirror exposure $[F(5,75)=9.28$, $\mathrm{p}<.001]$. Also, hours of mirror exposure interacted significantly with combat experience $[F(10,75)=2.03$, $\mathrm{p}<.05$; see Figure 3]; displays decreased quickly between 0 and $24 \mathrm{~h}$ in the aggressive combat group, but increased between 0 and $8 \mathrm{~h}$ and between 24 and $48 \mathrm{~h}$ in the nonaggressive group.

There also were significant main effects of combat experience $[F(2,15)=3.98, p<.05]$ and of hours of mirror viewing $[F(5,75)=4.15, p<.01]$ for the duration of avoidance behavior measure (facing $180 \mathrm{deg}$ away from mirror reflection). These data are presented in Figure 4. As can be seen, the initial avoidance behavior durations were similar for all three groups; however, by the end of $24 \mathrm{~h}$ of mirror viewing, fish in the no-combat group were avoiding their mirror reflections for much longer periods of time than fish in the aggressive- and nonaggressiveexperience groups.

Combat testing. The combat tests for the aggressiveand nonaggressive-experience groups were analyzed in two ways: (1) Comparisons were made between the three combat tests conducted prior to mirror viewing, and (2) comparisons were made between the means of these three combat tests and the combatretest scores. Both sets of data were subjected to ANOVA, with combat experience as an independent factor and combat tests as a repeated factor. Analyses of the first three combat tests indicated that fish attacked aggressive fish significantly more than they did nonaggressive opponents $[\mathrm{F}(1,10)=8.41, \mathrm{p}<.05]$ but did not display to them at significantly different rates $[F(1,10)<1, p>.05]$ or with significantly different display durations $[F(1,10)=1.18, p>.05]$.

A comparison of the number of attacks and the duration of displays during the three combat tests and the combat retest is presented in Figure 5. ANOVA performed on the duration-of-display data indicated that there was no significant main effect of combat experience $[F(1,10)=1.95, p>.05]$. The duration of displays, however, decreased significantly during the combat retest (following mirror viewing) $[\mathrm{F}(1,10)$ $=12.6, \mathrm{p}<.01$; see Figure 5a].

ANOVA performed on the attack data revealed a significant main effect of combat experience $[F(1,10)$ $=8.48, \mathrm{p}<.05]$; overall, aggressive opponents were attacked more than nonaggressive ones (see Figure 5b). In addition, the number of attacks decreased significantly during the combat retest $[\mathrm{F}(1,10)=6.91$, $\mathrm{p}<.05]$.
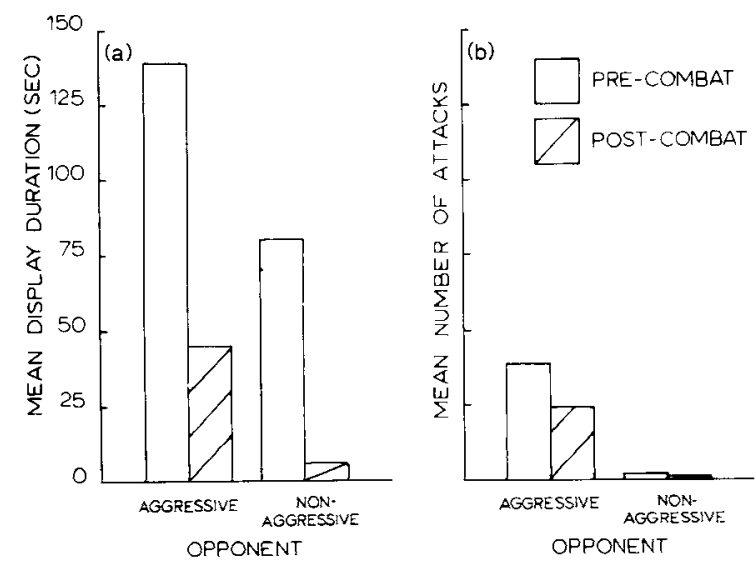

Figure 5. Mean display durations (a) and the mean number of attacks (b) directed towards the stimulus opponent by fish in the aggressive- and nonaggressive-experience groups. Data are shown for combat encounters prior to and following $96 \mathrm{~h}$ of mirror viewing. Scores for the combat tests prior to mirror viewing are based on three paired encounters, whereas the combat retest scores (following mirror viewing) are based on a single paired encounter. 


\section{DISCUSSION}

The findings of these two studies clarify the effects of prior social experience on the initiation and maintenance of Betta agonistic behavior. Specifically, Experiment 1 showed that fish that eventually became dominant displayed more often than their subordinate opponents before dominance was established, although both eventually dominant and subordinate fish attacked at similar rates until submission occurred. Dominant fish also showed a decrease in attacks, but not displays, once dominance was established. Preference test data indicated that subordinate fish were less inclined to view either a nonaggressive or aggressive conspecific than were more dominant fish. Experiment 2 demonstrated that fish attack aggressive opponents more than nonaggressive ones, although they display to them equally. Also, Experiment 2 showed that display and avoidance behavior durations wane at different rates as a function of prior combat experience. Fish in the nocombat-experience group avoided their reflections more than fish in the socially experienced groups, and the display durations of fish in the nonaggressiveexperience group waned less rapidly than those of fish in the other two groups.

These results support previous notions that visual signals and social responsiveness of the opponent can act to facilitate or inhibit Betta aggression (Dore et al., 1978; Johnson \& Johnson, 1973). In particular, the paired-encounter data from Experiments 1 and 2 suggest that submissive and nonaggressive behaviors inhibit attacks by Betta; male Betta attack aggressive opponents more than nonaggressive ones and rarely attack opponents once they have submitted. These results contradict the findings of Baenninger (1968), which suggest that submissive behaviors do little to suppress attacks and displays by dominant opponents. The contradictory results are best attributable to differences in the size of testing tanks, since Baenninger's data were based on combat testing in very small containers (1 liter), whereas ours ranged from 3.8 to 28.5 liters. Evidence in support of this interpretation comes from studies that suggest that fights in smaller tanks occur more often (Cain, Jessen, \& Flanagan, 1980) and last for longer periods of time than do those in larger tanks (Goldstein, 1975).

Although submissive and nonaggressive behaviors of the opponent appear to inhibit attacks, they do not have the same effect on displays. Dominant fish do not show a significant decrease in displays once their opponent has submitted (Experiment 1), nor do fish display differentially to aggressive and nonaggressive opponents (Experiment 2). These findings complement the literature on tank size and Betta aggression. For example, it has been shown that the inverse relationship found between tank size and the number of attacks does not hold for displays (Cain et al., 1980; Goldstein, 1975). A common feature of the social behavior and tank size situations appears to be whether it is necessary to aggress; both an opponent who appears nonthreatening and a tank size that is large enough to allow for escape result in a significant decrease in fighting behavior. However, fish continue to display in both situations. If it is assumed that displaying is more energetically efficient and potentially less aversive than attacking, then it follows that displaying might continue as a means of signaling readiness to fight, thereby maintaining dominance.

The results of the present study also provide data on the effects of prior social experience on noncombat agonistic situations. The finding, in Experiment 1 , that subordinate fish are less inclined to view conspecifics than are more dominant fish, is consistent with the results of others. For example, when dominant and subordinate fish were permitted to view either familiar or unfamiliar opponents, Meliska et al. (1975) reported that dominant fish spent more time approaching and displaying to their visual opponents than did subordinate fish. Also, Baenninger (1970) reported that dominant fish performed at a higher operant level than submissive fish when reinforcement was the opportunity to view a mirror.

The results of Experiment 2 suggest that the prior combat experience of fish affects "habituation" of the threat display following prolonged periods of mirror exposure. If one simply looks at the no-combat group, the predictable curve (e.g., Clayton \& Hinde, 1968) for waning of displays is evident. However, if fish are given combat experience prior to mirror exposure, several interesting relationships emerge. For example, the display durations of fish that have had prior combat experience with an aggressive opponent are initially much longer than those of the nonaggressiveand no-combat-experience groups; by $96 \mathrm{~h}$, all three groups have similarly low display durations. In addition, an increase in display duration after $8 \mathrm{~h}$ is evident for fish in the nonaggressive-experience group. This increase may reflect the fact that fish in the nonaggressive group must learn that their "opponent" (the mirror) will display back. That is, these fish initially may have a reduced proclivity to display as a result of predicting no displays from their nonaggressive opponents in the three previous combat encounters. Such an interpretation is consistent with the findings of Rhoad et al. (1975), which suggest that the waning of displays might reflect the fact that a fish may come to associate its stimulus opponent with a lack of consequences for displaying.

Finally, the mirror-viewing data indicate that avoidance interpretations of the waning of Betta threat displays may apply only to situations in which fish have no obvious prior social experience. It had previously been suggested that the attenuation of 
Betta displays reflects an increased avoidance of the opponent (Bols, 1977; Rhoad et al., 1975). Results of Experiment 2 demonstrate that fish that have had combat experience with either an aggressive or nonaggressive opponent do not show an increase in avoidance behavior as their display durations wane. Fish in the no-combat-experience group spent, on the other hand, approximately $25 \%$ of each observation session facing $180 \mathrm{deg}$ away from the mirror. Along with the display and attack data, these results emphasize the necessity of controlling for prior social experience when investigating the initiation and maintenance of Betta agonistic behaviors.

\section{REFERENCES}

BaEnninger, R. Catechol amines and social relations in Siamese fighting fish. Animal Behaviour, 1968, 16, 442-447.

BAENNINGER, R. Visual reinforcement, habituation, and prior social experience of Siamese fighting fish. Journal of Comparative and Physiological Psychology, 1970, 71, 1-5.

BaEnNinger, R. Some consequences of aggressive behavior: A selective review of the literature on other animals. Aggressive Behavior, 1974, 1, 17-37.

Bots, R. J. Display reinforcement in the Siamese fighting fish. Betta splendens: Aggressive motivation or curiosity. Journal of Comparative and Physiological Psychology, 1977, 91, 233-244.

Cain, N. W., Jessen, C., \& Flanagan, M. Social responsiveness and physical space as determinants of agonistic behavior in Betta splendens. Animal Learning \& Behavior, 1980, 8, 497-501.

Clayton, F. L., \& Hinde, R. A. The habituation and recovery of aggressive display in Betta splendens. Behaviour, 1968, 30, 96-106.

Dore, F., Lefebvre, L., \& Ducharme, R. Threat display in Betta splendens: Effects of water condition and type of agonistic stimulation. Animal Behaviour, 1978, 26, 738-745.

Goldstein, S. R. Observations on the establishment of a stable community of adult male and female Siamese fighting fish
(Betta splendens). Animal Behaviour, 1975, 23, 179-185.

Johnson, R. N., \& Johnson, L. D. Intra- and interspecific social and aggressive behavior in the Siamese fighting fish, Betta splendens. Animal Behaviour, 1973, 21, 665-672.

Leshner, A. I., \& Nock, B. L. The effects of experience on agonistic responding: An expectancy theory interpretation. Behavioral Biology, 1976, 17, 561-566.

Lовв, M. L., \& MCCAIN, G. Procedurally related differences in the aggressive behavior of Betta splendens (Regan). Animal Learning \& Behavior, 1976, 4, 367-373.

Mel.ISKa, J. A., \& Meliska, C. J. Effects of habituation on threat display and dominance establishment in the Siamese fighting fish, Betta splendens. Animal Learning \& Behavior. 1976, 4, 167-171.

Meliska, J., Meliska, C., Hoyenga, K., Hoyenga, K., \& WARD, E. Approach tendency and threat display as related to social status of Siamese fighting fish, Betta splendens. Animal Learning \& Behavior, 1975, 3, 135-139.

Miley, W. M., \& Burack, G. Strength of aggressive display in Siamese fighting fish (Betta splendens) toward a conspecific, an alien species (Macropodus opercularis), and a mirror image as affected by prior conspecific visual experience. Behavioral Biology, 1977, 21, 267-272.

Peeke, H. V. S., \& Peeke, S. C. Habituation of conspecific aggression responses in the Siamese fighting fish (Betto splendens). Behaviour, 1970, 36, 232-245.

Rhoad, K. D., Kalat, J. W., \& Klopfer, P. H. Aggression and avoidance by Betta splendens towards natural and artificial stimuli. Animal Learning \& Behavior, 1975, 3, 271-276.

\section{NOTE}

1. Three of the 13 pairs of fish failed to meet the criterion-fordominance establishment by the end of the seventh encounter and, therefore, were not included in the preference test. Also, due to unexpected mortality, four of the original 13 pairs of fish had to be dropped from the preference tests.

(Received for publication September 25, 1979; revision accepted March 23, 1980.) 